\title{
VARIABILIDADE ESPACIAL DE MICRONUTRIENTES EM SOLO SOB PIVÔ CENTRAL NO SUL DO ESTADO DE MATO GROSSO ${ }^{1}$
}

\author{
EDUARDO GUIMARÃES COUTO² e EGON KLAMT ${ }^{3}$
}

\begin{abstract}
RESUMO - Este estudo foi desenvolvido para identificar, caracterizar e comparar a estrutura da dependência espacial dos micronutrientes boro, cobre, ferro, manganês e zinco solúveis em um Latossolo Vermelho-Escuro sob pivô central após 14 anos de uso intensivo, no sul do Estado de Mato Grosso. O esquema de amostragem consistiu de coletas de 132 amostras com espaçamento regular de $167 \mathrm{~m}$, especialmente idealizado para determinar a variabilidade espacial em distância de até $1 \mathrm{~m}$. Com exceção do zinco, o uso intensivo propiciou um aumento significativo nas concentrações desses nutrientes na camada mais afetada pelo manejo $(0-20 \mathrm{~cm})$, mesmo assim insuficientes para atingir o nível crítico estabelecido para a região. Cerca de $95 \%$ das amostras de boro, $75 \%$ das amostras de cobre, $95 \%$ das amostras de manganês e 1,5\% das amostras de zinco apresentaram valores abaixo do nível crítico, distribuídos diferentemente pelos quadrantes, o que mostra que as práticas de fertilização e/ou as operações de preparo de solo não foram eficientes na distribuição e homogeneização dos fertilizantes.
\end{abstract}

Termos para indexação: geoestatística, agricultura de precisão.

\section{SPATIAL VARIABILITY OF MICROELEMENTS IN SOIL UNDER CENTER PIVOT IRRIGATION SYSTEM IN SOUTHERN MATO GROSSO STATE, BRAZIL}

\begin{abstract}
This study was carried out to identify, characterize and compare spatial structures of boron, copper, iron, manganese and zinc in a Dark-Red Latosol under crop field intensively managed for 14 years with a center pivot irrigation system in the Southern Mato Grosso State, Brazil. The soil sampling scheme, consisting of 132 systematic sampling, in which sampling points were located at regular intervals of $167 \mathrm{~m}$, in such a way that it was possible to determine the soil variability at $1 \mathrm{~m}$ distance. The result showed that, with exception of the zinc, the intensive land use increased significantly the nutrients concentration in the upper layer, however it was insufficient to reach the critical level established for the region. About $95 \%$ of boron samples, $75 \%$ of copper samples, $95 \%$ of manganese samples and $1.5 \%$ of zinc samples presented values below the critical level. These values were differently distributed by the quadrants in the center pivot, showed that fertilizers application and /or soil tillage were not efficient to distribute and to homogenize the fertilizers.
\end{abstract}

Index terms: geostatistics, precision agriculture.

\section{INTRODUÇÃO}

Por muitos anos presumiu-se que para um determinado solo as propriedades físicas e químicas

\footnotetext{
${ }^{1}$ Aceito para publicação em 24 de novembro de 1998.

${ }^{2}$ Eng. Agr., Dr., Prof. Adjunto, Dep. de Solos e Engenharia Rural, Univ. Federal do Mato Grosso, CEP 78060-900 Cuiabá, MT. E-mail: ecouto@zaz.com.br

${ }^{3}$ Eng. Agr., Ph.D., Prof. Titular, Dep. de Solos, Universidade Federal do Rio Grande do Sul, Caixa Postal 776, CEP 90001-970 Porto Alegre, RS. E-mail: fklamt@zaz.com.br
}

relacionadas a determinada profundidade poderiam ser expressas através da média e variância a partir de um número suficientemente grande de amostras. Este tipo de enfoque, baseado na estatística clássica, supõe que a variabilidade de determinada propriedade associada à sua média é aleatória e não contém referência à distribuição espacial das diferenças, dentro de uma mesma mancha de solo (Trangmar et al., 1985). No entanto, vários trabalhos empregando técnicas da geoestatística têm mostrado que a variabilidade do solo não é aleatória, e apresenta 
dependência espacial (Trangmar et al., 1985; Webster, 1985; Viloria, 1989; Vieira \& Maria, 1996).

A geoestatística tem mostrado grande utilidade para caracterizar e mapear este tipo de variação, com os mais diversos propósitos (Bouma et al., 1989; Fromm et al., 1993; Martinez \& Zinck, 1994; Moglen \& Bras, 1995; Schlesinger et al.,1996). Os procedimentos mais comuns incluem a caracterização e o modelamento da variabilidade espacial, por meio da estimativa dos valores nos locais não visitados, simulações espaciais, e otimização dos esquemas de amostragens (Cambardella et al., 1994).

Nos últimos 15 a 20 anos, a geoestatística tem sido aplicada em muitos trabalhos que avaliaram a variabilidade do solo, porém com poucas aplicações na área de química do solo (Davis et al., 1995), e mais restrita ainda é a sua aplicação na variabilidade espacial de microelementos (Zhang et al., 1995).

No Brasil, Albuquerque et al. (1996) aplicaram esta técnica para estudar a variabilidade espacial da espessura do horizonte A e do teor de argila e da produtividade da cultura do milho em um Podzólico Vermelho-Amarelo, em Santa Maria, RS, e encontraram em todas as variáveis estudadas dependência espacial superior a 70\%. Também no Rio Grande do Sul, Berg \& Klamt (1997), estudando a variabilidade espacial de características de solos do Planalto Médio, encontraram que a maior variância, no tocante ao $\mathrm{pH}$ a curtas distâncias, estava relacionada à distribuição não-homogênea de $\mathrm{Ca}$ e à grande variância entre glebas na quantidade de corretivo utilizada pelos produtores.

Souza et al. (1997), estudando a variabilidade de propriedades físicas e químicas do solo de um pomar cítrico, em Mangabeira, BA, verificaram que, com exceção da saturação de bases e Al, as demais propriedades mostraram dependência espacial, com alcance variando de $18 \mathrm{~m}$ (areia total e argila) a 59 m (matéria orgânica, $\mathrm{Ca}, \mathrm{Mg}, \mathrm{Ca}+\mathrm{Mg}$ e soma de bases).

O boro (B), o cobre $(\mathrm{Cu})$, o manganês $(\mathrm{Mn})$ e o zinco $(\mathrm{Zn})$ são os elementos em mais baixa disponibilidade nos solos de cerrado, e afetam drasticamente as espécies neles cultivadas (Sfredo et al., 1997).

Procurou-se, neste trabalho, identificar, caracterizar e comparar a distribuição da estrutura espacial de alguns micronutrientes (boro, cobre, manganês e zin- co solúveis) em solo sob pivô central após 14 anos de uso intensivo.

\section{MATERIAL E MÉTODOS}

A área de estudo compreende um pivô central de 170 ha cultivado intensivamente nos últimos 14 anos (Tabela 1), sendo composta da associação de Latossolo Vermelho-Escuro álico, textura argilosa, e de Latossolo Vermelho-Amarelo álico, textura média, em relevo plano e suave ondulado.

O esquema de amostragem consistiu de coletas de 132 amostras, com espaçamento regular especialmente idealizado para captar a variabilidade espacial em distância de até um metro (Fig. 1). As amostras de solo, após secadas ao ar e peneiradas foram analisadas de acordo com os procedimentos apresentados por Tedesco et al. (1985). O manganês foi extraído com $\mathrm{KCl} 1 \mathrm{M}$; o ferro, com oxalato de amônio ((NH4) $)_{2} \mathrm{C}_{2} \mathrm{O}_{4} \cdot \mathrm{H}_{2} \mathrm{O}$ pH 3,0) 0,2 M; e o cobre e o zinco, com $\mathrm{HCl} 0,1 \mathrm{M}$, e determinados por espectrofotometria de absorção atômica. O B foi extraído com água quente e determinado por colorimetria.

A análise estatística foi realizada em duas etapas. Primeiramente, aplicou-se a estatística clássica, para identificar os efeitos do uso da terra nas propriedades estudadas. A estatística descritiva (média, mínimo, máximo, e coeficiente de variação), o teste t e os testes de normalidade foram realizados por meio do programa Systat (Wilkinson, 1992).

TABELA 1. Descrição sumarizada do histórico da área experimental nos últimos 10 anos de uso agrícola ${ }^{1}$.

\begin{tabular}{|c|c|c|c|}
\hline Cultura/ano & Fertilizantes $\left(\mathrm{kg} \mathrm{ha}^{-1}\right)$ & $\begin{array}{l}\text { Produtividade } \\
\left(\mathrm{kg} \mathrm{ha}^{-1}\right)\end{array}$ & $\begin{array}{l}\text { Calagem } \\
\left(\mathrm{kg} \mathrm{ha}^{-1}\right)\end{array}$ \\
\hline Milho/1 & $\begin{array}{l}400(3-30-16+Z n) \\
160 \text { (Uréia) }\end{array}$ & 5.100 & 2.000 \\
\hline Soja/2 & $300(0-20-20)$ & 2.760 & - \\
\hline Trigo/3 & $\begin{array}{l}400(3-30-16+Z n) \\
100 \text { (Uréia) }\end{array}$ & 2.100 & - \\
\hline Soja/4 & $300(0-20-20)$ & 2.640 & - \\
\hline Feijão/5 & $\begin{array}{l}300(0-20-20) \\
50 \text { (Uréia) }\end{array}$ & 1.380 & 2.000 \\
\hline Soja/6 & $300(0-20-20)$ & 1.990 & - \\
\hline Soja/7 & $300(0-20-20)$ & 2.520 & - \\
\hline Feijão/8 & $\begin{array}{l}300(0-20-20) \\
50 \text { (Uréia) }\end{array}$ & 1.290 & 2.500 \\
\hline Arroz/9 & $\begin{array}{l}174(0-20-20+Z n) \\
50 \text { (Uréia) }\end{array}$ & 900 & - \\
\hline Soja/10 & $\begin{array}{l}300(0-20-20) \\
60(\mathrm{KCl}) \\
700(\text { Yorin })\end{array}$ & 1.080 & - \\
\hline Milho/10 & $400(5-25-25)$ & $-{ }^{1}$ & \\
\hline
\end{tabular}




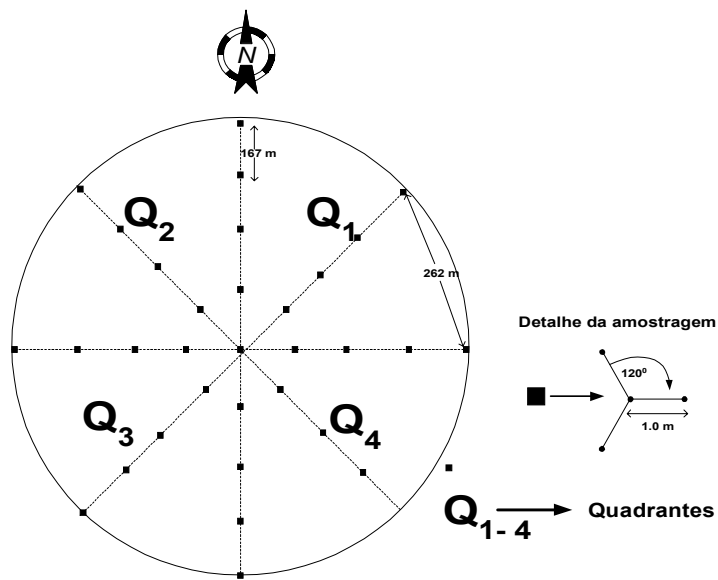

FIG. 1. Esquema de amostragem na área do pivô central.

Embora esses métodos estatísticos permitam inferir sobre a variabilidade do solo (vertical e horizontal), a dependência espacial entre as amostras somente pode ser modelada através da geoestatística.

Sabe-se que os solos e muitas outras propriedades da superfície da terra mudam de uma parte para outra, dentro de uma área. Porém, as propriedades podem, usualmente, ser reconhecidas como estacionárias dentro de uma pequena vizinhança - por exemplo de um tamanho V. Esta situação é chamada de quase-estacionariedade em $\mathrm{z}(\mathrm{x})-\mathrm{z}(\mathrm{x}+\mathrm{h})$. Isto leva ao seguinte modelo de variação (Webster \& Oliver, 1990):

$\mathrm{Z}(\mathrm{x})=\mu_{\mathrm{v}}+\varepsilon(\mathrm{x})$

onde $\mathrm{Z}(\mathrm{x})$ é o valor da propriedade $\mathrm{Z}$ para $\mathrm{x}$ dentro da região $\mathrm{V} ; \mu_{\mathrm{v}}$ é o valor médio dentro da região; e $\varepsilon(\mathrm{x})$ é o componente aleatório com uma média zero e variância definida como:

$\operatorname{var}[\varepsilon(\mathrm{x})-\varepsilon(\mathrm{x}+\mathrm{h})]=\mathrm{E}\left[\{\varepsilon(\mathrm{x})-\varepsilon(\mathrm{x}+\mathrm{h})\}^{2}\right]=2 \gamma(\mathrm{h})$.

$\mathrm{Na}$ prática, limita-se o tamanho da área sobre a qual fazemos nossas estimativas; por esta razão, é usado o subscrito $V$ na equação da página anterior.

Neste estudo, a análise da dependência espacial foi feita através de semivariogramas, com base nas pressuposições de estacionariedade da hipótese intrínseca, o qual pode ser estimado por:

$\hat{\gamma}(h)=\frac{1}{2 N(h)} \sum_{i=1}^{N(h)}\left[z\left(s_{i}\right)-z\left(s_{i}+h\right)\right]^{2}$, onde $\mathrm{N}(\mathrm{h})$ é o número de pares de pontos $\mathrm{z}\left(\mathrm{s}_{\mathrm{i}}\right)$ e $\mathrm{z}\left(\mathrm{s}_{\mathrm{i}}+\mathrm{h}\right)$, separados por um vetor $\mathrm{h}$ através do qual os dois pontos $\mathrm{s}_{\mathrm{i}}$ e $\mathrm{s}_{\mathrm{i}+\mathrm{h}}$. O semivariograma é caracterizado por três propriedades: o efeito pepita $\left(\mathrm{C}_{0}\right)$, o patamar $(\mathrm{C})$ e o alcance (a). O efeito pepita $\left(\mathrm{C}_{0}\right)$ é a descontinuidade do semivariograma próximo à origem: $\lim _{\mathrm{h} \rightarrow 0} \gamma(\mathrm{h})=\mathrm{C}_{0}$, e expressa tanto a variabilidade para uma escala menor que o intervalo de amostragem quanto à variação não-espacial. O efeito pepita não pode ser removido pela proximidade das amostragens, mas apenas ser mensurado por esta medida (Trangmar et al., 1985; Webster, 1985; Cahn et al., 1994). O patamar, quando existe, ocorre a partir da distância onde a variância aproxima-se assintoticamente do máximo; o alcance expressa a distância além da qual as amostras não são correlacionadas. O semivariograma depende tanto da magnitude quanto da direção de $\mathrm{h}$. As propriedades do solo são isotrópicas quando elas variam de maneira similar em todas as direções, no caso de o semivariograma depender apenas da distância entre as amostras, h. A anisotropia geométrica ocorre quando as variações para a distância $h$ em uma direção são equivalentes às variações $\mathrm{h}$ em outras direções.

Os semivariogramas, além de fornecerem informações a respeito da anisotropia e medidas de correlação entre as distâncias, h, podem ser usados no estimador e na variância de Krigagem, que é um procedimento não-estocástico de interpolação que apresenta estimadores não tendenciosos e de variância mínima (Webster, 1985). Leva ainda em consideração o número de amostras utilizadas, as posições das amostras na área a ser avaliada, as distâncias entre as amostras e a zona a ser estimada e a continuidade espacial da variável em estudo (Andriotti, 1988). O estimador de Krigagem é do tipo BLUE ("best linear unbiased estimator”), que é expresso matematicamente pela equação: $\mathrm{Zk}=\sum_{\mathrm{i}=1}^{\mathrm{n}} \lambda_{\mathrm{i}} \cdot \mathrm{Z}\left({ }_{\mathrm{x}_{\mathrm{i}}}\right)$, a qual representa uma combinação linear dos dados, onde i representa os ponderadores e $\mathrm{z}_{(\mathrm{xi})}$ os dados experimentais.

A formulação do estimador de Krigagem em termos de um modelo linear tem sido de grande valor para aumentar sua transparência (Stein, 1995b). O uso da Krigagem permite atingir um dos objetivos centrais dos estudos sobre variabilidade espacial, que é obter, a partir de observações pontuais, informações para grandes áreas de terra, tais como campos de cultivo, baseadas nas observações da variável a ser estimada em locais não amostrados (Stein, 1995a; Voltz et al., 1997).

Além de se estimar valores ou variâncias das propriedades espacialmente distribuídas, há um interesse crescente em determinar curvas de probabilidade através da Krigagem disjuntiva e da Krigagem de indicadores múltiplos (quando na ausência de tendências), o que permite determinar as probabilidades de um indicador ambiental exceder um nível crítico (Finke, 1992; Stein, 
1995a; 1995b). Neste estudo, os níveis críticos utilizados foram os determinados por Lopes (1984) e Galrão (1996) em solos de cerrado, e as estimativas das probabilidades foram feitas através do programa DISCO (Finke \& Stein, 1992) e expressas através do software Surfer (Keckler, 1997).

Os semivariogramas foram estimados por meio dos programas SPATANAL e SPATIN desenvolvidos por Stein \& Staritsky (1993), e modelados experimentalmente pelo programa WLSFIT (Heuvelink, 1993). Para descrever os semivariogramas e fornecer informações mais precisas para Krigagem, os semivariogramas experimentais foram avaliados pela técnica de cross-validation, que permite comparar o impacto dos diferentes modelos de semivariogramas sobre os resultados da interpolação (Goovaerts, 1997).

Neste trabalho, os modelos foram escolhidos a partir dos valores do erro do quadrado médio (MSE) da variabilidade dependente do espaço (VDE) e da relação $\mathrm{SSD} / \mathrm{SST} \leq 0,50$. O MSE compara cada estimativa com cada observação e é definido como a média dos erros estimados ao quadrado; para um procedimento realístico, seu valor nunca é zero, mas deve ser pequeno. A VDE é obtida da seguinte equação: $\mathrm{VDE}=100-100\left(\mathrm{C}_{\mathrm{o}} / \mathrm{C}_{\mathrm{o}}+\mathrm{C}\right)$, onde $\mathrm{C}_{\mathrm{o}}+\mathrm{C}$ é o patamar, quando a $\mathrm{VDE}$ for $\geq 50 \%$. A relação $\mathrm{SSD} / \mathrm{SST}$ é a razão entre a soma dos quadrados dos desvios com a soma dos quadrados totais e é usada para quantificar o ajuste do modelo do semivariograma; um ajuste próximo a zero dá um ajuste excelente e um valor próximo a um dá um ajuste muito limitado.

\section{RESULTADOS E DISCUSSÃO}

Um sumário dos parâmetros da estatística descritiva consta na Tabela 2. Os resultados mos- traram que, com exceção do $\mathrm{Zn}$, o uso intensivo propiciou um aumento significativo nas concentrações destes nutrientes na camada mais afetada pelo manejo (0-20 cm), mesmo assim insuficientes (com exceção do B, cujo nível crítico não foi estabelecido) para atingir o nível crítico estabelecido para a região (Lopes, 1984).

Um exame mais detalhado das distribuições de freqüência associadas aos níveis críticos de cada propriedade no tocante à profundidade de $0-20 \mathrm{~cm}$ no pivô central, mostrou que, com exceção do $\mathrm{Zn}$, houve deficiência localizada, em todas as propriedades estudadas (Fig. 2). Cerca de 95\% das amostras de B, $75 \%$ das amostras de $\mathrm{Cu}$ e $95 \%$ das amostras de $\mathrm{Mn}$ apresentaram valores abaixo do nível crítico, distribuídos diferentemente pelos quadrantes, o que sugere que as práticas de fertilização, apesar de pontualmente apresentarem valores elevados (expressos pelos valores máximos apresentados na Tabela 2), não foram suficientes. Além disso, as operações de preparo do solo parecem não ter sido eficientes para distribuir e homogeneizar os fertilizantes aplicados (Fig. 2).

A razão para os baixos valores apresentados parece estar muito mais relacionada com práticas de fertilização insuficientes, do que com problemas na química de solo destes elementos, pois grande parte do $\mathrm{B}$, por exemplo, preso à matéria orgânica, pode estar sendo afetado pelo uso intensivo do solo, sendo liberado, após a mineralização, para a solução do solo (Dantas, 1991). A prática da calagem aumenta sua disponibilidade, atingindo valores máximos na faixa de $\mathrm{pH} 5,5-6,5$ (Dantas, 1991). Os valores

TABELA 2. Estatística descritiva e nível crítico das amostras estudadas (132 observações).

\begin{tabular}{crllccccc}
\hline Propriedade & Prof. $(\mathrm{cm})$ & Unidade & Média & Mínimo & Máximo & CV (\%) & Nível crítico \\
\hline Boro & $0-20$ & $\mathrm{mg} \mathrm{kg}^{-1}$ & $0,37^{*}$ & 0,20 & 0,60 & 33 & $0,5^{1}$ \\
& $20-40$ & & 0,31 & 0,10 & 0,60 & 34 & \\
Cobre & $0-20$ & $\mathrm{mg} \mathrm{kg}^{-1}$ & $0,73^{*}$ & 0,30 & 3,40 & 55 & $0,8^{1}$ \\
& $20-40$ & & 0,43 & 0,10 & 0,90 & 28 & \\
\multirow{2}{*}{ Manganês } & $0-20$ & $\mathrm{mg} \mathrm{kg}^{-1}$ & $3,00^{*}$ & 1,00 & 7,00 & 48 & $5,0^{2}$ \\
& $20-40$ & & 1,66 & 1,00 & 4,00 & 40 & & \\
\multirow{2}{*}{ Zinco } & $0-20$ & $\mathrm{mg} \mathrm{kg}^{-1}$ & $7,29 *$ & 0,80 & 21,00 & 50 & $1,0^{3}$ \\
& $20-40$ & & 0,90 & 0,10 & 8,50 & 128 & & \\
\hline
\end{tabular}

${ }^{1}$ Lopes \& Guilherme (1984).

${ }^{2}$ Lopes (1984).

${ }^{3}$ Galrão (1996).

* Médias diferem significativamente nas profundidades pelo teste t a $0,1 \%$. 
de $\mathrm{pH}$ encontrados neste estudo ( $\overline{\mathrm{x}}=5,4$ e $\mathrm{CV}=6 \%$ ) são compatíveis com a faixa de máxima absorção.

$\mathrm{O} \mathrm{Cu}$, da mesma forma que o $\mathrm{B}$, está relacionado à bioacumulação. Em solos cultivados por certo tempo, com a matéria orgânica mais estável, como deve ser o caso do pivô central em estudo, a calagem provoca a diminuição do $\mathrm{Cu}$, uma vez que os complexos da matéria orgânica com o elemento apresentam maior estabilidade em valores de $\mathrm{pH}$ mais elevados (Ferreira \& Cruz, 1991). Além disso, há redução na solubilidade do elemento, na forma de óxidos, com aumento na quantidade adsorvida sobre as superfícies ou precipitada (Harsem \& Vlek, 1985, citados por Ferreira \& Cruz, 1991). Novamente, o valor médio de pH de 5,4 é insuficiente para limitar a disponibilidade do $\mathrm{Cu}$.

$\mathrm{O} \mathrm{Mn}$, da mesma forma, apresenta maior disponibilidade em $\mathrm{pH}$ mais baixo. A deficiência em
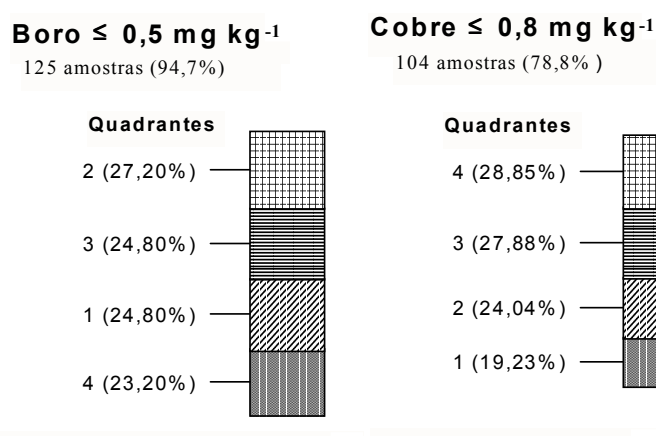

Cobre $\leq 0,8 \mathrm{mg} \mathrm{kg}^{-1}$ 104 amostras $(78,8 \%)$

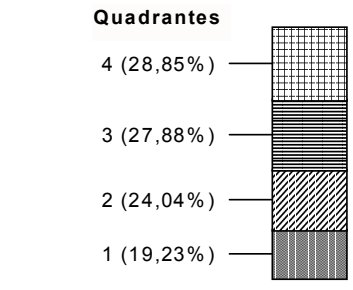

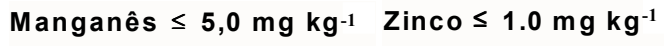
$125 \operatorname{amostras}(94,7 \%)$ 2 amostras $(1,5 \%)$
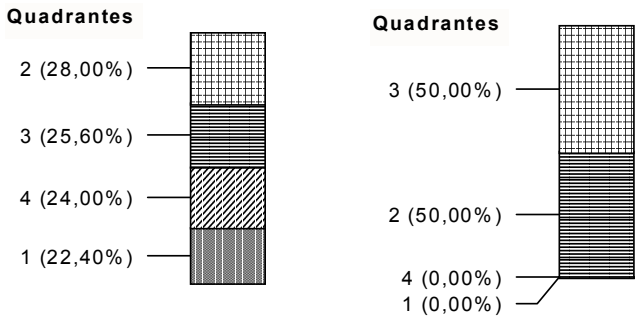

FIG. 2. Amostras e os quadrantes associados no solo sob pivô central abaixo do nível crítico estabelecido para os solos da região.
Mn ocorre quando os valores de $\mathrm{pH}$ são superiores a 6,2 (Borkert, 1991), mas o mesmo autor alerta para o fato de que uma série de outros fatores pós-coleta (secagem das amostras ao ar, tempo de secagem) influem na sua disponibilidade.

Considerando que o manejo do solo tem grande influência na variabilidade destas propriedades, os valores do CV apresentados na Fig. 3 expressam esta magnitude. $\mathrm{Cu}, \mathrm{Mn}$ e $\mathrm{Zn}$ tiveram CVs acima de $50 \%$, e em alguns casos, os CVs se mantiveram elevados mesmo na profundidade de $20-40 \mathrm{~cm}$, alcançando até $128 \%$, como no caso do $\mathrm{Zn}$.

Estes valores de CVs são semelhantes aos encontrados em outros estudos (Western, 1978;
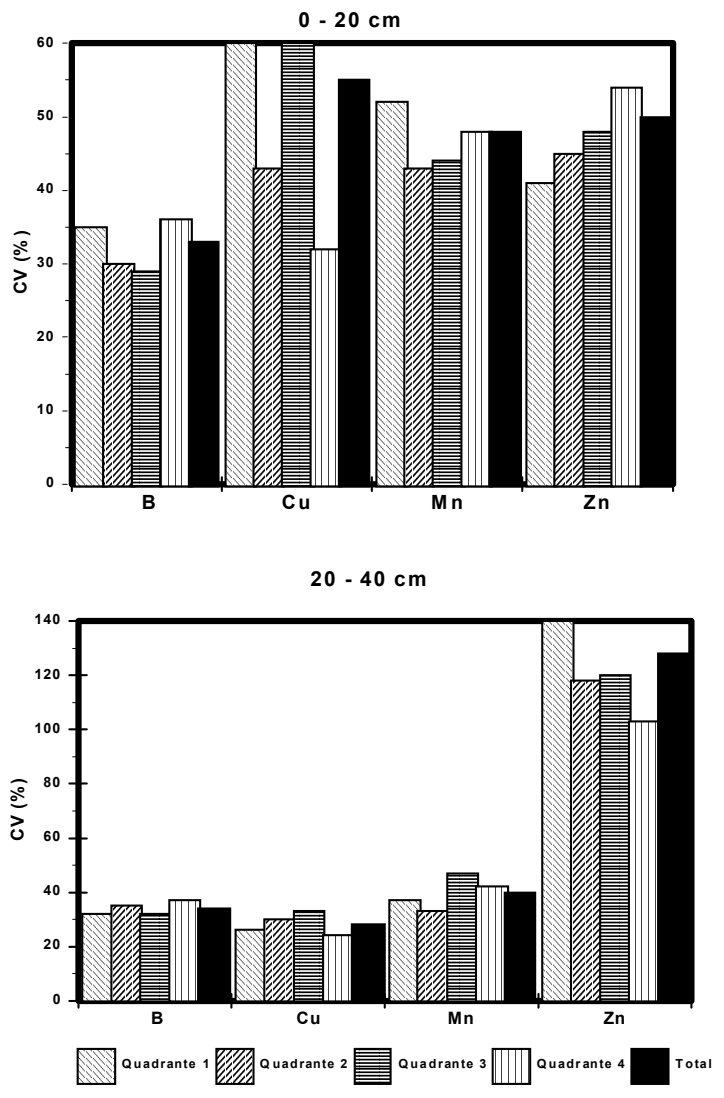

FIG. 3. Coeficiente de variação (CV) das propriedades associadas às situações estudadas no pivô central.

Pesq. agropec. bras., Brasília, v.34, n.12, p.2321-2329, dez. 1999 
Wilding, 1985; Souza, 1992; Dobermann, 1994; Davis et al., 1995), e as práticas de preparo do solo, como por exemplo, o uso de subsolador, parecem aumentar a variação destas propriedades nas áreas de cultivo, com grande efeito inclusive na camada subsuperficial. Os quadrantes apresentaram valores diferenciados de CVs, sendo que os menores valores de $\mathrm{CVs}$ foram encontrados nos quadrantes 4 $(0-20 \mathrm{~cm})$ e $1(20-40 \mathrm{~cm})$, e os maiores valores, nos quadrantes $1(0-20 \mathrm{~cm})$ e $2(20-40 \mathrm{~cm})$.

Este fato denota um comportamento diferencial do uso, e sugere que nem todo o pivô é cultivado simultaneamente, isto é, diferentes operações de preparo de solo e distribuição de fertilizantes são realizadas ao longo do tempo e do espaço, dentro dos diferentes quadrantes (que nada mais são do que uma divisão arbitrária do pivô).

Os valores médios dos $\mathrm{CV}$ s referentes às profundidades de 0-20 e 20-40 cm foram, respectivamente, $41 \%$ e $50 \%$, o que denota grande variabilidade em ambas as profundidades. Esta grande variação nas concentrações dos micronutrientes estudados tem influência na estrutura espacial das propriedades, conforme mostra a Tabela 3.

Os resultados mostraram que o $\mathrm{B}$ (nas duas profundidades) e o $\mathrm{Cu}$ (na profundidade de $0-20 \mathrm{~cm}$ ) não apresentaram dependência espacial. Considerando os critérios estabelecidos (VDE $\geq 50 \%$ e $\mathrm{SSD} / \mathrm{SST} \leq 0,50$ ), apenas o Mn apresentou estrutura espacial bem definida, com alcance da dependência de $385 \mathrm{~m}$ na profundidade de $0-20 \mathrm{~cm}$, e de $412 \mathrm{~m}$ na profundidade de $20-40 \mathrm{~cm}$.
A partir dos parâmetros do semivariograma do Mn na profundidade de 0-20 cm (Fig. 4), o qual apresentou o melhor ajuste entre os micronutrientes estudados (Tabela 3), determinouse a probabilidade de encontrar valores que excedessem determinado nível crítico através da cokrigagem disjuntiva. Os resultados da Fig. 5, exemplificados para o caso do Mn, mostraram que a deficiência deste micronutriente foi generalizada, o que foi também verificado no campo, onde plantas de milho apresentaram sintoma de deficiência deste elemento.

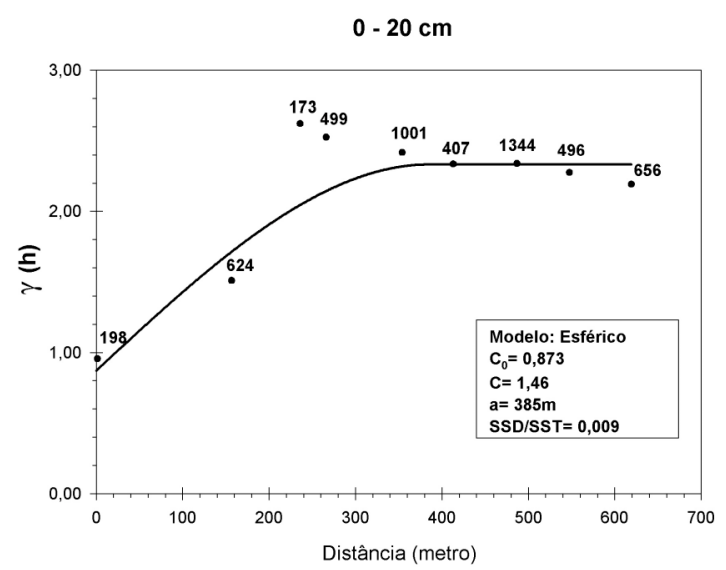

FIG. 4. Semivariograma do manganês para a profundidade de $0-20 \mathrm{~cm}$.

TABELA 3. Parâmetros dos semivariogramas estudados.

\begin{tabular}{lrlllllll}
\hline Propriedade & Prof. $(\mathrm{cm})$ & Modelo & $\mathrm{C}_{\mathrm{o}}$ & $\mathrm{C}$ & Patamar & VDE (\%) & Alcance (m) & SSD/SST \\
\hline Boro & $0-20$ & Efeito. Pepita puro & & & & & & \\
& $20-40$ & Efeito. Pepita puro & & & & & & \\
Cobre $^{1}$ & $0-20$ & Efeito. Pepita puro & & & & & & \\
& $20-40$ & Exponencial & 0,010 & 0,010 & 0,020 & 43 & 132 & 0,71 \\
Manganês & $0-20$ & Esférico & 0,873 & 1,460 & 2,333 & 63 & 385 & 0,09 \\
Zinco $^{1}$ & $20-40$ & Exponencial & 0,250 & 0,330 & 0,570 & 57 & 412 & 0,30 \\
${\text { Zinco }(\log )^{1}}^{1}$ & $0-20$ & Gaussiano & 0,024 & 0,014 & 0,038 & 37 & 548 & 0,72 \\
\hline
\end{tabular}

${ }^{1}$ Semivariogramas descartados (VDE $\leq 50 \%$ e SSD/SST $\geq 0,50$ ). 


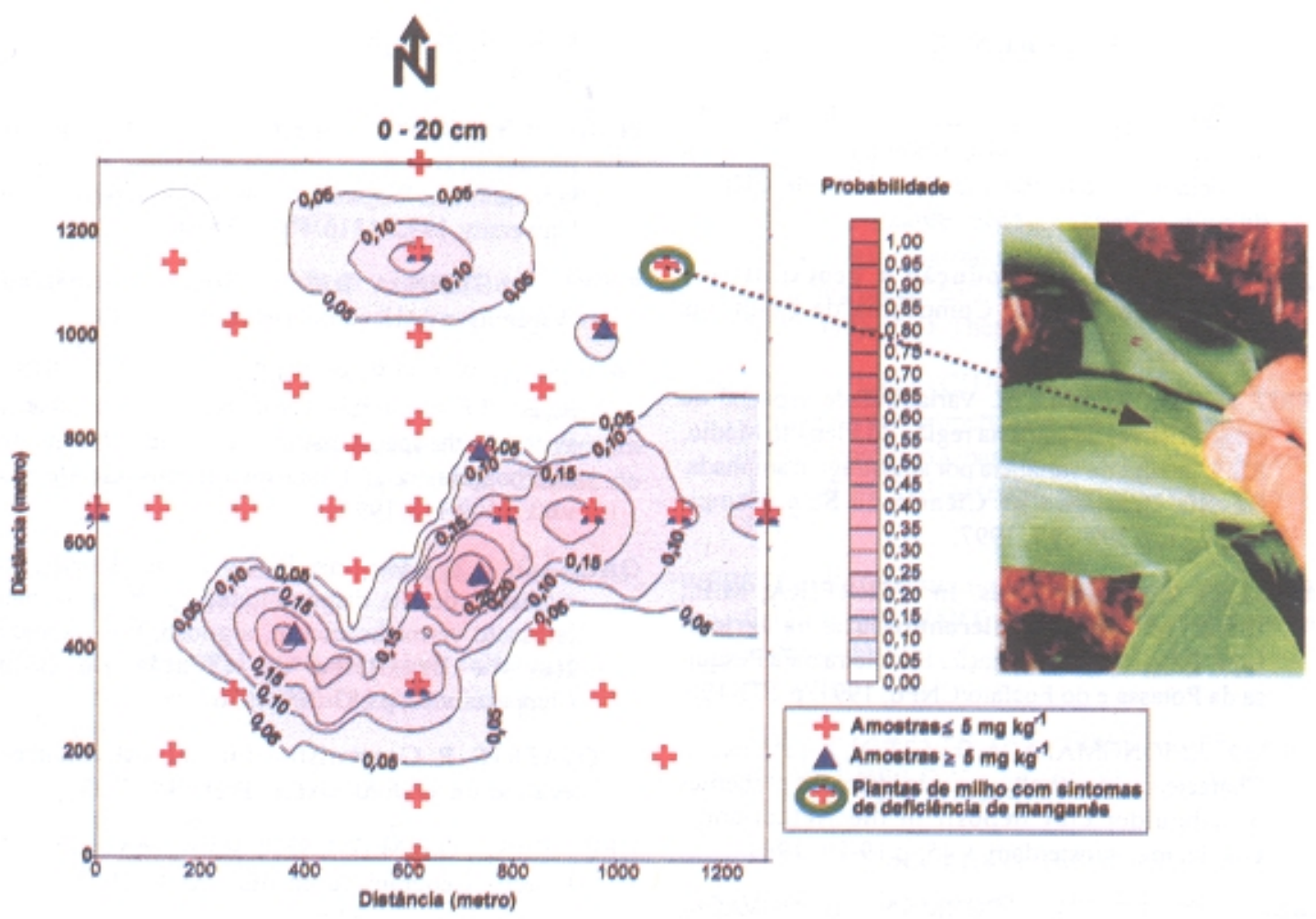

FIG. 5. Probabilidade do manganês solúvel exceder a $5,0 \mathrm{mg} \mathrm{kg}^{-1}$ no solo sob pivô central na profundidade de $0-20 \mathrm{~cm}$, através da cokrigagem disjuntiva, com destaque para planta de milho com sintoma de deficiência deste elemento.

\section{CONCLUSÕES}

1. A deficiência localizada dos micronutrientes estudados deve-se à quantidade insuficiente de adubos aplicada, considerando que os valores médios, com exceção do zinco, estão abaixo dos níveis críticos.

2. Os altos valores dos coeficientes de variação em todos os micronutrientes estudados indicam que as práticas de fertilização e as operações de preparo de solo não são eficientes na distribuição e homogeneização dos fertilizantes nos quadrantes do pivô central.

3. O alcance da dependência espacial, em geral, aumenta com a profundidade, sugerindo que as práticas de manejo executadas ao longo dos anos interferem na estrutura espacial destas propriedades.

4. A interpolação por Krigagem permite estimar os efeitos espaciais impostos pelas práticas de manejo naquelas propriedades que apresentam estrutura espacial bem definidas, viabilizando uma aplicação diferencial de corretivos, otimizando o uso dos insumos e minorando os problemas ambientais advindos do uso da terra, na busca de uma agricultura de precisão.

\section{AGRADECIMENTOS}

Ao engenheiro agrônomo Wilson José de Souza, pelas informações referentes ao histórico da área experimental.

Pesq. agropec. bras., Brasília, v.34, n.12, p.2321-2329, dez. 1999 


\section{REFERÊNCIAS}

ALBUQUERQUE, J.A.; REINERT, D.J.; FIORIN, J.E. Variabilidade de solo e planta em podzólico vermelho-amarelo. Revista Brasileira de Ciência do Solo, v.20, p.151-157, 1996.

ANDRIOTTI, J.L.S. Introdução à geoestatística linear. Porto Alegre: Companhia de Pesquisas Minerais, 1988. 99p.

BERG, M.V.D.; KLAMT, E. Variabilidade espacial de características de solos na região do Planalto Médio, RS: I. análise da variância por amostragem aninhada. Revista Brasileira de Ciência do Solo, Campinas, v.21, p.393-399, 1997.

BORKERT, C.M. Manganês. In: FERREIRA, M.E.; CRUZ, M.C. (Eds.). Micronutrientes na agricultura. Piracicaba: Associação Brasileira para Pesquisa da Potassa e do Fosfato/CNPq, 1991. p.173-190.

BOUMA, J.; JONGMANS, A.G.; STEIN, A.; PEEK, G. Characterizing spatially variable hydraulic properties of a boulder clay deposit in the Netherlands. Geoderma, Amsterdam, v.45, p.19-29, 1989.

CAMBARDELLA, C.A.; MOORMAN, T.B.; NOVACK, J.M.; PARKIN, T.B.; KARLEN, D.L.; TURCO, R.F.; KNOPKA, A.E. Field-scale variability of soil properties in central Iowa soils. Soil Science Society of America. Journal, Madison, v.58, p.1240-1248, 1994.

CAHN, M.D.; HUMMEL, J.W.; BROER, B.H. Spatial analysis of soil fertility for site-specific crop management. Soil Science Society of America Journal, Madison, v.58, p.1240-1248, 1994.

DANTAS, J.P. Boro. In: FERREIRA, M.E.; CRUZ, M.C.P. (Eds.). Micronutrientes na agricultura. Piracicaba: Associação Brasileira para Pesquisa da Potassa e do Fosfato/CNPq, 1991. p.113-130.

DAVIS, J.G.; HOSSNER, L.R.; WILDING, L.; MANU, A. Variability of soil chemical properties in two sandy, dunal soils of Niger. Soil Science, Baltimore, v.159, p.321-330, 1995.

DOBERMANN, A. Factors causing field variation of direct-seeded flooded rice. Geoderma, Amsterdam, v.62, p.125-150, 1994.

FERREIRA, M.E.; CRUZ, M.C.P. Cobre. In: FERREIRA, M.E.; CRUZ, M.C.P. (Eds.). Micronutrientes na agricultura. Piracicaba: Associação Brasileira para
Pesquisa da Potassa e do Fosfato/CNPq, 1991. p.131-157.

FINKE, P. Spatial variability of soil structure and its impact on transport process and some associated land qualities. Wageningen: Wageningen Agricultural University, 1992. 131p. Ph.D. Thesis.

FINKE, A.; STEIN, A. DISCO software. Wagening: Wageningen Agricultural University, 1992.

FROMM, H.; WINTER, K.; FILSER, J.; HANTSCHEL, R.; BEESE, F. The influence of soil type and cultivation system on the spatial distribution of the soil fauna and microorganisms and their interactions. Geoderma, v.60, p.109-118, 1993

GALRÃO, E.Z. Métodos de aplicação de zinco e avaliação de sua disponibilidade para milho num latossolo vermelho-escuro, argiloso, fase Cerrado. Revista Brasileira de Ciência do Solo, Campinas, v.20, p.283-289, 1996.

GOOVAERTS, P. Geostatistics for natural resources evaluation. Oxford: Oxford Press, 1997. 483p.

HEUVELINK, G.B.M. WLSFIT software version 3.5. Utrecht: Geographical Institute RUU, 1993.

KECKLER, D. Surfer 6.0 for windows. User's guide. New York: Golden Software, 1997.

LOPES, A.S. Solos sob "Cerrado": características, propriedades e manejo. Piracicaba: Associação Brasileira para a Pesquisa da Potassa e do Fosfato, 1984. 62p.

LOPES, A.S.; GUILHERME, L.R.G. Solos sob cerrado: manejo da fertilidade para a produção agropecuária. São Paulo: Associação Nacional para Difusão de Adubos e Corretivos Agrícolas, 1984. 47p. (Boletim técnico, 5).

MARTINEZ, L.J.; ZINCK, J.A. Modelling spatial variations of soil compaction in the Guaviare colonization area, Colombian Amazonia. ITC Journal, Enschede, v.3, p.252-263, 1994.

MOGLEN, G.E.; BRAS, R.L. The importance of spatially heterogeneous erosivity and the cumulative area distribution within a basin evolution model. Geomorphology, Amsterdam, v.12, p.173-185, 1995.

SCHLESINGER, W.H.; RAIKES, J.A.; HARTLEY, A.E.; CROSS, A.F. On the spatial pattern of soil nutrients in desert ecosystems. Ecology, v.77, p.364-375, 1996. 
SFREDO, G.S.; BORKERT, C.M.; NEPOMUCENO, A.L.; OLIVEIRA, M.C. N. Eficácia de produtos contendo micronutrientes, aplicados via semente, sobre produtividade e teores de proteína da soja. Revista Brasileira de Ciência do Solo, Campinas, v.21, p.41-45, 1997.

SOUZA, L.S. Variabilidade espacial do solo em sistemas de manejo. Porto Alegre: UFGRS, 1992. 166p. Tese de Doutorado.

SOUZA, L.D.S.; COGO, N.P.; VIEIRA, S.R. Variabilidade de propriedades físicas e químicas do solo em um pomar cítrico. Revista Brasileira de Ciência do Solo, v.21, p.367-372, 1997.

STEIN, A. Spaces for data. ITC Journal, Enschede, v.3, p.261-265, 1995a.

STEIN, A. Spatial statistics for soils and the environment: Preliminary version. Enschede: ITC, 1995b. 72p.

STEIN, A.; STARITSKY, I. G. SPATANAL , SPATIN and OPTIM Software. Wageningen: Wageningen Agricultural University, 1993.

TEDESCO, M.J.; VOLKWEISS, S.J.; BOHNEN, H.; GIANELLO, C.; BISSANI, C. Análise de solos, plantas e outros materiais. Porto Alegre: UFRGS, 1985. 215p.

TRANGMAR, B.B.;YOST, R.S.; UEHARA, G. Applications of geostatistics to spatial studies of soil properties. Advances in Agronomy, Orlando, v.38, p.45-94, 1985.

VIEIRA, S.; MARIA, I.C. de. Delineamento experimental e análise estatística na pesquisa em conservação do solo. In: CASTRO FILHO, C.C.;
MUZILLI, O. (Eds.). Manejo integrado de solos em microbacias hidrográficas. Londrina: IAPAR/ Socidade Brasileira de Ciência do Solo, 1996. p.257-268.

VILORIA, J.A. The inter-relation between soil survey and soil information systems: optimization of data gathering procedures. Oxford: University of Oxford, 1989.350p. PhD. Thesis.

VOLTZ, M.; LAGACHERIE, P.; LOUCHART, X. Predicting soil properties over a region using sample information from a mapped reference area. European Journal of Soil Science, Oxford, v.48, p.19-30, 1997.

WEBSTER, R. Quantitative spatial analysis of soil in the field. In: STEWART, B.A. (Ed.). Advance in soil science. New York: Springer-Verlag, 1985. v.3, p. $1-70$.

WEBSTER, R.; OLIVER, M.A. Statistical methods in soil and land resource survey. Oxford: Oxford University Press, 1990. 316p.

WESTERN, S. Soil survey contracts and quality control. Oxford: Clarendon Press, 1978. 284p.

WILDING, L. Spatial variability: its documentation accommodation and implication to soil surveys. In: NIELSEN, D.R.; BOUMA, J. (Eds.). Soil spatial variability. Wageningen: Pudoc, 1985. p.166-194.

WILKINSON, L. Systat for Windows. Evanston: Systat, 1992. $547 \mathrm{p}$.

ZHANG, R.; RAHMAN, S.; VANCE, G.F.; MUNN, L.C. Geostatistical analysis of trace elements in soils and plants. Soil Science, Baltimore, v.159, p.383-390, 1995. 